https://helda.helsinki.fi

Optimization of numerical orbitals using the Helmholtz kernel

\author{
Solala, Eelis
}

2017-02-28

Solala , E , Losilla , S A , Sundholm , D , Xu , W \& Parkkinen , P 2017 , ' Optimization of numerical orbitals using the Helmholtz kernel ', Journal of Chemical Physics, vol. 146 , no. 8,084102 . https://doi.org/10.1063/1.4976557

http://hdl.handle.net/10138/307281

https://doi.org/10.1063/1.4976557

publishedVersion

Downloaded from Helda, University of Helsinki institutional repository.

This is an electronic reprint of the original article.

This reprint may differ from the original in pagination and typographic detail.

Please cite the original version. 


\section{Optimization of numerical orbitals using the Helmholtz kernel}

Eelis Solala, Sergio A. Losilla, Dage Sundholm, Wenhua Xu, and Pauli Parkkinen

Citation: The Journal of Chemical Physics 146, 084102 (2017); doi: 10.1063/1.4976557

View online: http://dx.doi.org/10.1063/1.4976557

View Table of Contents: http://aip.scitation.org/toc/jcp/146/8

Published by the American Institute of Physics

\section{Articles you may be interested in}

Improved method for generating exchange-correlation potentials from electronic wave functions

The Journal of Chemical Physics 146, 084103084103 (2017); 10.1063/1.4975990

Nonlocality of relaxation rates in disordered landscapes

The Journal of Chemical Physics 146, 084104084104 (2017); 10.1063/1.4976844

The escape problem for mortal walkers

The Journal of Chemical Physics 146, 084106084106 (2017); 10.1063/1.4976522

Outstanding performance of configuration interaction singles and doubles using exact exchange Kohn-Sham orbitals in real-space numerical grid method

The Journal of Chemical Physics 145, 224309224309 (2016); 10.1063/1.4971786

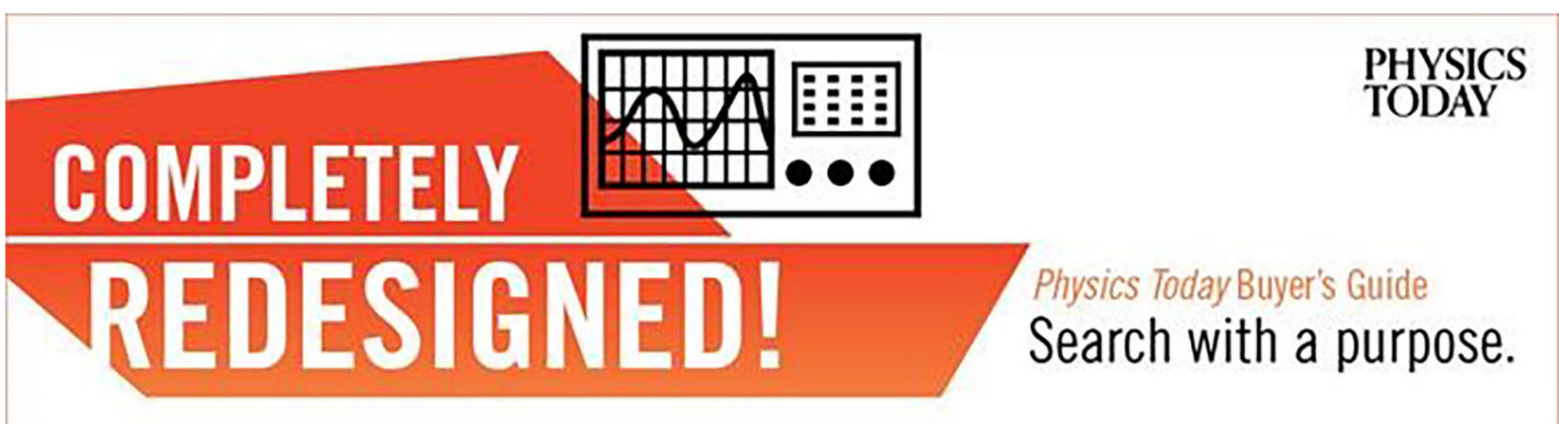




\title{
Optimization of numerical orbitals using the Helmholtz kernel
}

\author{
Eelis Solala, Sergio A. Losilla, Dage Sundholm, Wenhua Xu, and Pauli Parkkinen \\ Department of Chemistry, University of Helsinki, P.O. Box 55, A.I. Virtanens plats 1, \\ FIN-00014 Helsinki, Finland
}

(Received 13 October 2016; accepted 2 February 2017; published online 22 February 2017)

\begin{abstract}
We present an integration scheme for optimizing the orbitals in numerical electronic structure calculations on general molecules. The orbital optimization is performed by integrating the Helmholtz kernel in the double bubble and cube basis, where bubbles represent the steep part of the functions in the vicinity of the nuclei, whereas the remaining cube part is expanded on an equidistant threedimensional grid. The bubbles' part is treated by using one-center expansions of the Helmholtz kernel in spherical harmonics multiplied with modified spherical Bessel functions of the first and second kinds. The angular part of the bubble functions can be integrated analytically, whereas the radial part is integrated numerically. The cube part is integrated using a similar method as we previously implemented for numerically integrating two-electron potentials. The behavior of the integrand of the auxiliary dimension introduced by the integral transformation of the Helmholtz kernel has also been investigated. The correctness of the implementation has been checked by performing Hartree-Fock self-consistent-field calculations on $\mathrm{H}_{2}, \mathrm{H}_{2} \mathrm{O}$, and $\mathrm{CO}$. The obtained energies are compared with reference values in the literature showing that an accuracy of $10^{-4}$ to $10^{-7} E_{h}$ can be obtained with our approach. Published by AIP Publishing. [http://dx.doi.org/10.1063/1.4976557]
\end{abstract}

\section{INTRODUCTION}

The time-independent Schrödinger equation for bound states

$$
\left(-\frac{1}{2} \nabla^{2}+V(\mathbf{r})\right) \psi(\mathbf{r})=\epsilon \psi(\mathbf{r})
$$

can be formulated as an integral equation by first reorganizing it into a general Helmholtz equation

$$
\left(\nabla^{2}+2 \epsilon\right) \psi(\mathbf{r})=2 V(\mathbf{r}) \psi(\mathbf{r}),
$$

the Green's function equation of which has to be solved iteratively because the wavefunction $\psi(\mathbf{r})$ occurs on both sides of the equation and the energy eigenvalue $(\epsilon<0)$ of the bound state is also not known and has to be updated for each guess for $\psi(\mathbf{r})$. The kinetic energy is given by $-\frac{1}{2} \nabla^{2} \psi(\mathbf{r})$ and $V(\mathbf{r})$ is the potential. To maintain the familiar Green's function notation, we denote $k=\sqrt{-2 \epsilon}$. The Green's function can then be written as ${ }^{1-11}$

$$
\begin{aligned}
\psi(\mathbf{r}) & =\left(\nabla^{2}+2 \epsilon\right)^{-1} 2 V(\mathbf{r}) \psi(\mathbf{r}) \\
& =-\frac{1}{2 \pi} \int \frac{\exp \left(-k\left|\mathbf{r}-\mathbf{r}^{\prime}\right|\right)}{\left|\mathbf{r}-\mathbf{r}^{\prime}\right|} V\left(\mathbf{r}^{\prime}\right) \psi\left(\mathbf{r}^{\prime}\right) d \mathbf{r}^{\prime},
\end{aligned}
$$

where $\mathbf{r}$ and $\mathbf{r}^{\prime}$ represent the three Cartesian coordinates $x$, $y, z$. The solution of Eq. (3) is then obtained iteratively by integrating

$$
\psi(\mathbf{r})=2 \int G\left(\mathbf{r}, \mathbf{r}^{\prime}\right) V\left(\mathbf{r}^{\prime}\right) \psi\left(\mathbf{r}^{\prime}\right) d \mathbf{r}^{\prime},
$$

where $G\left(\mathbf{r}, \mathbf{r}^{\prime}\right)$ denotes the Green's function and $\psi(\mathbf{r})$ is the wave function or orbital that vanishes at the boundaries of the integration domain. The energy eigenvalue can obtained in each iteration by calculating the expectation value for the
Hamiltonian or by using another approach that yields an estimated value for the energy eigenvalue. To our knowledge, this approach was employed for the first time in 1962 by Kalos, ${ }^{12}$ who formulated a Helmholtz kernel approach for solving time-independent Schrödinger equations for nuclei by using the Monte Carlo integration. In this Helmholtz kernel approach, the differential equation is recast into an integral expression involving additional dimensions showing similarities with the Coulomb integral of two-electron electrostatic interactions.

In this work, we have implemented an approach to optimize numerical one-particle wave functions of bound electronic states by integrating the Helmholtz kernel using a similar approach as we previously employed for calculating electrostatic potentials using the Poisson kernel, i.e., integrating the Coulomb integral. ${ }^{13-17}$ The singular attraction potential between the electrons and the nuclei of molecular systems leads to very steep cusps of the orbitals and potentials in the vicinity of the nuclei. Since the steep cusps are difficult to expand using numerical methods, we have devised the use of a double numerical basis, where the steep parts of the functions are expanded in one-center functions at each nucleus, whereas the remainder is described using a three-dimensional (3D) equidistant grid. ${ }^{16}$ The $3 \mathrm{D}$ numerical functions are expanded in an outer product of local numerical functions in one dimension (1D). Our long-term goal is to develop fully numerical electronic structure methods that consider all electrons and scale well on parallel computers. Tensorial numerical methods have also been developed by other research groups for expanding functions on 3D grids. ${ }^{7,8,18-28}$ Tensorial based wavelet methods have been developed for calculating electrostatic potentials and solving electronic structure equations using the same approach. $1,2,5,7,11,23,29-39$ 
The article begins in Section II with a description of the double basis of the bubbles and cube framework for representing scalar functions. Then, we explain how the Helmholtz kernel approach fits into the numerical framework. The main features of the numerical integration of the Helmholtz kernel in the double basis are outlined in Section IV. The shape of the $\mathrm{t}$-space integrand and the t-space integration is discussed in Section V. The orbital optimization procedure, the HartreeFock iterative scheme, is sketched in Section V. The method is demonstrated by calculating Hartree-Fock energies for a number of small molecules and compared them to accurate literature values in Section VI.

\section{NUMERICAL REPRESENTATION OF SCALAR FUNCTIONS}

Scalar functions such as orbitals, potentials, electron density, and energy density are expanded in the double numerical basis, where the steep part of the functions in the vicinity of the nuclei is expanded in a sum of atom centered numerical radial functions multiplied with spherical harmonics. The onecenter functions are called bubbles. The difference between the sum of the one-center bubble functions and the total function is expanded using the 3D tensorial grid functions leading to a numerical representation whose accuracy can be systematically increased towards the basis-set limit by using denser grids. The functions expanded on the 3D grid are called cube. ${ }^{16}$ A given 3D function $f(\mathbf{r})$ is expanded in the double basis as

$$
f(\mathbf{r})=f^{\Delta}(\mathbf{r})+\sum_{A=1}^{N_{A}} f^{A}\left(r_{A}, \theta_{A}, \varphi_{A}\right),
$$

where $f^{\Delta}(\mathbf{r})$ is the cube, $f^{A}\left(r_{A}, \theta_{A}, \varphi_{A}\right)$ are the bubble functions, and $r_{A}, \theta_{A}$, and $\varphi_{A}$ are spherical coordinates centered at the point nucleus of atom $\mathrm{A}\left(R_{A}\right)$. The bubble functions representing the steep part of the functions render accurate all-electron calculations feasible using an equidistant cube grid. The bubble functions are separated into a radial part $f^{A l m}\left(r_{A}\right)$ and an angular part consisting of real spherical harmonics $Y_{l m}\left(\theta_{A}, \varphi_{A}\right)$. We use Racah's normalization of the spherical harmonics in this work,

$$
f^{A}\left(r_{A}, \theta_{A}, \varphi_{A}\right)=\sum_{l=0}^{l_{\max }} \sum_{m=-l}^{l} f^{A l m}\left(r_{A}\right) Y_{l m}\left(\theta_{A}, \varphi_{A}\right) .
$$

The radial part of the bubble functions is divided into $M$ small intervals, where the function is expanded in a local basis consisting of seven regular Lagrange interpolating polynomials of sixth $(P)$ order, ${ }^{16,40}$

$$
\chi_{i}(x)=\prod_{l \neq i}^{P+1} \frac{\left(x-x_{l}\right)}{\left(x_{i}-x_{l}\right)} .
$$

The number of grid points in the radial range is $N=M P$ +1 because the grid points at the element boundaries belong to two adjacent intervals. Since the amplitude of the Lagrange interpolation functions is 1 in one grid point and 0 in the others, the radial functions can be then written as

$$
f^{A l m}\left(r_{A}\right)=\sum_{I=1}^{N} f_{I}^{A l m} \chi_{I}\left(r_{A}\right),
$$

where the expansion coefficient $f_{I}^{A l m}$ is the value of the radial function in grid point $I$ and $\chi_{I}\left(r_{A}\right)$ are the local element functions along the radial range. The number and length of radial elements depend on the nuclear charge of atom A. The elements are shorter close to the nucleus and their length increases farther away from the nucleus. The 1D radial grid can have several thousands of grid points.

The cube is expanded in a tensorial product of locally defined numerical 1D basis functions

$$
f^{\Delta}(r)=\sum_{i j k} f_{i j k}^{\Delta} \chi_{i}^{x}(x) \chi_{j}^{y}(y) \chi_{k}^{z}(z),
$$

where $f_{i j k}^{\Delta} \equiv f^{\Delta}\left(x_{i}, y_{j}, z_{k}\right)$ are the expansion coefficients corresponding to the value of the function at the grid points. The 1D Cartesian grids are equidistant and $\chi_{i}^{\xi}(\xi), \xi=x, y, z$ are Lagrange interpolation functions of order six. The size of the cube is typically $150-500$ grid points in each dimension. The bubbles and cube basis is discussed in more detail in Ref. 16.

\section{INTEGRATION OF THE POISSON KERNEL}

The electrostatic potential due to a given charge density $(\rho(\mathbf{r}))$ can be obtained by integrating the Poisson kernel in the bubbles and cube basis,

$$
\begin{aligned}
V(\mathbf{r}) & =\int_{\Omega} \frac{\sum_{A, l m} \rho^{A l m}\left(r_{A}^{\prime}\right) Y_{l m}\left(\theta_{A}^{\prime}, \phi_{A}^{\prime}\right)+\rho^{\Delta}\left(\mathbf{r}^{\prime}\right)}{\left|\mathbf{r}-\mathbf{r}^{\prime}\right|} \mathrm{d}^{3} r^{\prime} \\
& =\sum_{A, l m} V^{A l m}\left(r_{A}\right) Y_{l m}\left(\theta_{A}, \varphi_{A}\right)+V^{\Delta}(\mathbf{r}),
\end{aligned}
$$

using the shorthand notation above. The radial bubble functions are obtained by dividing the molecular density into atomic domains and projecting them onto the spherical harmonic functions using

$$
\rho^{A l m}\left(r_{A}\right)=\int_{0}^{2 \pi} \int_{0}^{\pi} \omega_{A}(\mathbf{r}) \rho(\mathbf{r}) Y_{l m}\left(\theta_{A}, \phi_{A}\right) \sin \left(\theta_{A}\right) \mathrm{d} \theta_{A} \mathrm{~d} \phi_{A},
$$

where $\omega_{A}(\mathbf{r})$ are masking functions. The projection yields the density contribution that is localized at nucleus A. ${ }^{16}$ The radial part of one-center potentials is calculated by using inward and outward integrations of the radial densities, whereas the cube part is obtained by a direct 3D numerical integration. ${ }^{13-15}$ For the integration of the Poisson kernel, the Coulomb operator is rewritten using the integral expression and discretized as

$$
\begin{aligned}
\frac{1}{\left|\mathbf{r}_{1}-\mathbf{r}_{2}\right|} & =\frac{2}{\sqrt{\pi}} \int_{0}^{\infty} \exp \left(-t^{2} r_{12}^{2}\right) \mathrm{d} t \\
& \approx \sum_{p}^{p_{\max }} \omega_{p} \exp \left(-t_{p}^{2} r_{12}^{2}\right)+\frac{\pi}{t_{f}^{2}} \delta\left(\mathbf{r}_{1}-\mathbf{r}_{2}\right) .
\end{aligned}
$$

The $t$-dependence of the Poisson kernel in Eq. (12) is integrated using quadrature in linear and logarithmic coordinates. From $t_{f}$ to infinity, the steep Gaussian is approximated as a Dirac delta function. $t_{p}$ and $\omega_{p}$ are the quadrature points and the integration weights, respectively. The final expression for the 
cube part of the Coulomb potential can be written as

$$
V_{i j k}^{\Delta}=\frac{2}{\sqrt{\pi}} \sum_{p}^{p_{\max }} \omega_{p} \sum_{k^{\prime}}^{N_{z}} F_{k k^{\prime}}^{z, p} \sum_{j^{\prime}}^{N_{y}} F_{j j^{\prime}}^{y, p} \sum_{i^{\prime}}^{N_{x}} F_{i i^{\prime}}^{x, p} \rho_{i^{\prime} j^{\prime} k^{\prime}}^{\Delta}+\frac{\pi}{t_{f}^{2}} \rho_{i j k}^{\Delta},
$$

where the matrix elements of $F_{i^{\prime} i}^{\xi, p}, \xi=x, y, z$ are calculated as $1 \mathrm{D}$ integrals over the local basis functions $\chi_{i}^{\xi}\left(\xi_{1}\right),{ }^{13-15}$

$$
F_{i i^{\prime}}^{\xi, p}=\int_{-\infty}^{\infty} \exp \left(-t_{p}^{2}\left(\xi_{i}-\xi_{1}\right)^{2}\right) \chi_{i^{\prime}}^{\xi}\left(\xi_{1}\right) \mathrm{d} \xi_{1} .
$$

Details of the bubbles and cube procedure are given in Ref. 16, where we also described how the double basis is used for accurate transformations of other Hamiltonian operators in numerical self-consistent-field calculations.

\section{INTEGRATION OF THE HELMHOLTZ KERNEL}

Integration of the Helmholtz kernel is a linear operation implying that it can also be performed separately for the bubble and the cube parts,

$$
\begin{aligned}
\int G\left(\mathbf{r}, \mathbf{r}^{\prime}\right) f\left(\mathbf{r}^{\prime}\right) d \mathbf{r}^{\prime}= & \int G\left(\mathbf{r}, \mathbf{r}^{\prime}\right) f^{\Delta}\left(\mathbf{r}^{\prime}\right) d \mathbf{r}^{\prime} \\
& +\sum_{A} \int G\left(\mathbf{r}, \mathbf{r}^{\prime}\right) f^{A}\left(\mathbf{r}^{\prime}\right) d \mathbf{r}^{\prime}
\end{aligned}
$$

In Eq. (15) $f^{\Delta}\left(\mathbf{r}^{\prime}\right)$ is a smooth function that is expanded on the $3 \mathrm{D}$ grid, whereas the steep part of the functions in the vicinity of the nuclei is expanded in the one-center functions at $R_{A}$. For the integration of the cube part, the singularity of the Green's function is avoided by introducing a similar integral transformation

$\frac{\exp \left(-k\left|\mathbf{r}-\mathbf{r}^{\prime}\right|\right)}{\left|\mathbf{r}-\mathbf{r}^{\prime}\right|}=\frac{2}{\sqrt{\pi}} \int_{0}^{\infty} \exp \left(-\frac{k^{2}}{4 t^{2}}-t^{2}\left|\mathbf{r}-\mathbf{r}^{\prime}\right|^{2}\right) d t$

for the Helmholtz kernel as used for the Poisson kernel. 3,7,11,13 The $t$ integration can be approximated by calculating the integral using quadrature

$$
\begin{aligned}
& \frac{2}{\sqrt{\pi}} \int_{0}^{\infty} \exp \left(-\frac{k^{2}}{4 t^{2}}-t^{2}\left|\mathbf{r}-\mathbf{r}^{\prime}\right|^{2}\right) d t \\
& \quad \approx \sum_{p} \omega_{p}^{\prime} \exp \left(-t_{p}^{2}\left|\mathbf{r}-\mathbf{r}^{\prime}\right|^{2}\right)+\frac{\pi}{t_{f}^{2}} \delta\left(\mathbf{r}-\mathbf{r}^{\prime}\right)
\end{aligned}
$$

with $\omega_{p}^{\prime}=\omega_{p} \exp \left(-\frac{k^{2}}{4 t_{p}^{2}}\right)$, where $\omega_{p}$ and $\omega_{p}^{\prime}$ are the integration weights of the Poisson and Helmholtz kernels, respectively, and $t_{p}$ are corresponding integration points. For large $t$ values, the integrand of the Helmholtz kernel has the same asymptotic form as the Poisson kernel because the $k$-dependent contribution to the integration weights is proportional to $t^{-2}$ in the exponent and declines rapidly for large $t$. The same integration method can be used for the Poisson kernel and the Helmholtz kernel, whose integration weights depend on the energy eigenvalue $\epsilon$ via $k$. Applying the operator in this form on $f^{\Delta}\left(\mathbf{r}^{\prime}\right)$, which in the Helmholtz case is the cube part of twice the product of the orbital $\psi\left(\mathbf{r}^{\prime}\right)$ multiplied with the potential $V\left(\mathbf{r}^{\prime}\right)$, whose expansion coefficients are $f_{i^{\prime} j^{\prime} k^{\prime}}^{\Delta}$. By integrating the first term on the right-hand-side of Eq. (15) over the $\mathbf{r}^{\prime}$ space, we obtain the final expression

$$
\psi_{i j k}^{\Delta}=\frac{2}{\sqrt{\pi}} \sum_{p}^{p_{\max }} \omega_{p}^{\prime} \sum_{k^{\prime}}^{N_{z}} F_{k k^{\prime}}^{z, p} \sum_{j^{\prime}}^{N_{y}} F_{j j^{\prime}}^{y, p} \sum_{i^{\prime}}^{N_{x}} F_{i i^{\prime}}^{x, p} f_{i^{\prime} j^{\prime} k^{\prime}}^{\Delta}+\frac{\pi}{t_{f}^{2}} f_{i j k}^{\Delta}
$$

for the expansion coefficients of the cube part of the orbitals $\left(\psi_{i j k}^{\Delta}\right)$. The expression for $F_{i i^{\prime}}^{\xi, p}$ is given in Eq. (14). ${ }^{13-16}$ The expansion coefficients $f_{i^{\prime} j^{\prime} k^{\prime}}^{\Delta}$ of the cube are obtained by projecting out the steep bubble contribution from $f(\mathbf{r})$ as described above for $\rho(\mathbf{r})$ in the case of the Poisson kernel. The methods of the projection are described in detail in Ref. 16.

The most convenient approach to the integration of the bubbles' part of the Helmholtz kernel is to expand the kernel in a formally infinite series of spherical harmonics multiplied with a modified spherical Bessel function of the first kind $\left(\hat{I}_{l+\frac{1}{2}}\left(k r_{<}\right)\right)$and a modified spherical Bessel function of the second kind $\left(\hat{K}_{l+\frac{1}{2}}\left(k r_{>}\right)\right)$, where $r_{<}$is the smaller of $r$ and $r^{\prime}$, whereas $r_{>}$is the larger of $r$ and $r^{\prime},{ }^{40}$

$$
\begin{aligned}
\frac{\exp \left(-k\left|\mathbf{r}-\mathbf{r}^{\prime}\right|\right)}{\left|\mathbf{r}-\mathbf{r}^{\prime}\right|}= & \frac{2 k}{\pi} \sum_{l=0}^{\infty}(2 l+1) \hat{I}_{l+\frac{1}{2}}\left(k r_{<}\right) \hat{K}_{l+\frac{1}{2}}\left(k r_{>}\right) \\
& \times \sum_{m=-l}^{l} Y_{l m}^{*}(\theta, \varphi) Y_{l m}\left(\theta^{\prime}, \varphi^{\prime}\right) .
\end{aligned}
$$

The expansion in Eq. (19) is analogous to the multipole moment expansion in the Coulomb case. For the bubble functions located at $R_{A}$, the integration of the $\mathbf{r}^{\prime}$ space of the Helmholtz kernel can then be written as

$$
\begin{aligned}
\int G\left(\mathbf{r}, \mathbf{r}^{\prime}\right) f^{A}\left(r_{A}^{\prime}, \theta^{\prime}{ }_{A}, \varphi_{A}^{\prime}\right) d \mathbf{r}^{\prime} \\
=8 k \sum_{l=0}^{l_{\max }} \sum_{m=-l}^{l} Y_{l m}\left(\theta_{A}, \varphi_{A}\right) \\
\quad \times \int_{0}^{\infty} r^{\prime 2} f^{A l m}\left(r^{\prime}\right) \hat{I}_{l+\frac{1}{2}}\left(k r_{<}\right) \hat{K}_{l+\frac{1}{2}}\left(k r_{>}\right) d r^{\prime},
\end{aligned}
$$

where the angular integrals over $\mathbf{r}^{\prime}$ are done analytically using the Racah's normalization of the spherical harmonics. The one dimensional integral can be further simplified to

$$
\begin{aligned}
\int_{0}^{\infty} r^{\prime 2} f^{A l m}\left(r^{\prime}\right) \hat{I}_{l+\frac{1}{2}}\left(k r_{<}\right) \hat{K}_{l+\frac{1}{2}}\left(k r_{>}\right) d r^{\prime} \\
=\hat{K}_{l+\frac{1}{2}}(k r) \int_{0}^{r} r^{\prime 2} f^{A l m}\left(r^{\prime}\right) \hat{I}_{l+\frac{1}{2}}\left(k r^{\prime}\right) d r^{\prime} \\
\quad+\hat{I}_{l+\frac{1}{2}}(k r) \int_{r}^{r_{\max }} r^{\prime 2} f^{A l m}\left(r^{\prime}\right) \hat{K}_{l+\frac{1}{2}}\left(k r^{\prime}\right) d r^{\prime}
\end{aligned}
$$

where $r_{\max }$ is the size of the radial range of the bubbles. The resulting radial functions of the bubbles $\left(g^{A l m}(r)\right)$ are obtained as

$$
\begin{aligned}
g_{A l m}(r)= & 8 k\left[\hat{K}_{l+\frac{1}{2}}(k r) \int_{0}^{r} r^{\prime 2} f^{A l m}\left(r^{\prime}\right) \hat{I}_{l+\frac{1}{2}}\left(k r^{\prime}\right) d r^{\prime}\right. \\
& \left.+\hat{I}_{l+\frac{1}{2}}(k r) \int_{r}^{r_{\max }} r^{\prime 2} f^{A l m}\left(r^{\prime}\right) \hat{K}_{l+\frac{1}{2}}\left(k r^{\prime}\right) d r^{\prime}\right],
\end{aligned}
$$


where the one-dimensional radial integration can be performed using quadrature. The long-range contribution to the cube part of the Helmholtz kernel can be calculated using a generalized fast multipole method involving the corresponding series expansion of spherical harmonics multiplied with the modified spherical Bessel function. ${ }^{41}$

\section{INTEGRATION IN THE T-SPACE}

The accuracy of the kernel integration of the cube part depends on the size of the Cartesian grid and the quality of the $t$ integration. The accuracy can be systematically improved by increasing the number of grid points. However, since the computational time increases linearly with the number of grid points of the $t$ integration, it is desirable to have as few integration points in the $t$ space as possible. Thus, the success of the $t$ integration depends on an optimal distribution of the integration points. In the Poisson kernel case, we divided the $t$-integration domain in three regions. The linear region $\left[0, t_{l}\right]$ is integrated using ordinary Gaussian quadrature, the $\left[t_{l}, t_{f}\right]$ region is integrated using Gaussian quadrature in logarithmic coordinates, and the integration in the interval of $\left[t_{f}, \infty\right]$ is calculated analytically leading to an error of the order of $t_{f}^{-4} \cdot{ }^{15}$ For the Helmholtz kernel, the shape of the $t$ integrand, i.e., the $F_{k}(t)$ function in Eq. (23) can be obtained from the normalization condition of ground-state hydrogenlike wave functions using different values for $k$ corresponding to the orbital energy, which is analogous to the approach we used when investigating the $t$ dependence of the Coulomb kernel, ${ }^{15}$

$$
\begin{aligned}
\int_{0}^{\infty} F_{k}(t) d t= & \frac{2}{\sqrt{\pi}} \int_{0}^{\infty}\left[\int \psi(\mathbf{r}) \int \exp \left(-\frac{k^{2}}{4 t^{2}}-t^{2}\left|\mathbf{r}-\mathbf{r}^{\prime}\right|^{2}\right)\right. \\
& \left.\times 2 V\left(\mathbf{r}^{\prime}\right) \psi\left(\mathbf{r}^{\prime}\right) d \mathbf{r}^{\prime} d \mathbf{r}\right] d t
\end{aligned}
$$

The calculations show that the shape of $F_{k}(t)$ is rather independent of $k$, implying that for reasonable values of the orbital energy $(\epsilon)$, the same $t$-integration grid can be used. However, the location of the maximum of the $t$ integrand depends slightly on $k$. The $F_{k}(t)$ function is shown for a number of $k$ values in Figure 1(a). By rescaling the variable $t$ to $s=\frac{t}{k}$, one obtains a practically general shape of the integrand that can be integrated numerically using the same integration grid for all values of $k$. Figure 1(b) shows the integrand after the change of variable. The orbital dependent $\exp \left(\frac{-k^{2}}{4 t^{2}}\right)$ factor takes the integrand rapidly to zero as $t \rightarrow 0$. For large $t$ values, the shape of $F_{k}(t)$ is the same as for the Coulomb kernel with the largest contribution to the integral from intermediate $t$ values. The main difference between $F_{k}(t)$ and the corresponding function in the Coulomb case appears at small $t$ values. However, since the atoms of the studied molecules are light, we have used the same $t$-integration grid for the Helmholtz and Poisson kernels in this work.

\section{ORBITAL OPTIMIZATION AND THE HARTREE-FOCK SCHEME}

The integration of the Helmholtz kernels for the different $k$ values, which are proportional to the square root of the approximate orbital energies, yields the updated orbitals. The right-hand-side of the kernel expression in Eq. (3) contains an effective potential due to the electron-electron and electron-nuclei interactions as described by Jensen et al. for Hartree-Fock level calculations. ${ }^{11}$ The individual contributions to the effective potential are calculated in the double basis as discussed in our previous works. ${ }^{13-16,42}$ All contributions to the Fock matrix can be calculated from the orbitals because the double basis approach renders accurate calculations of the kinetic energy contribution feasible as the bubbles remove the derivative discontinuity at the nuclei. ${ }^{16}$ The occupied-occupied part of the Fock matrix and the corresponding metric are constructed and diagonalized yielding orbital energies and canonical orbitals, since the application of the Helmholtz kernel does not necessarily conserve the orthogonality and the canonical properties of the orbitals. The occupied canonical orbitals are used in the calculation of the potentials that together with the orbital energies yield the updated orbitals when integrating the Helmholtz kernel. The convergence of the selfconsistent-field (SCF) iteration is accelerated by using the power method.

The SCF method is sketched in Algorithm 1, whereas the construction of the Fock matrix in the double basis is described in detail in Ref. 42. The Schrödinger equation can also be reorganized to a Poisson equation by adding the potential term to the energy on the right-hand-side. However, numerical tests showed that the resulting Poisson equation is not well conditioned leading to a slow convergence of the iterative scheme.

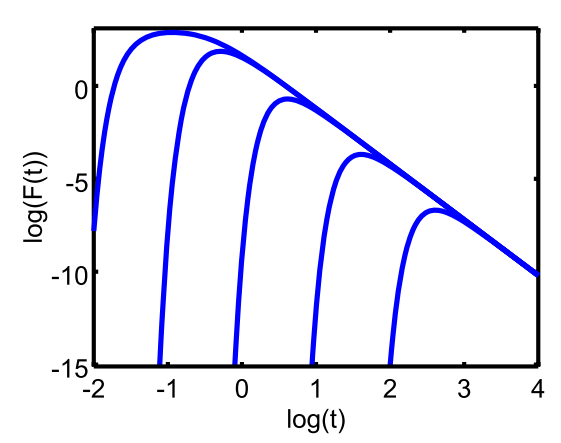

(a)

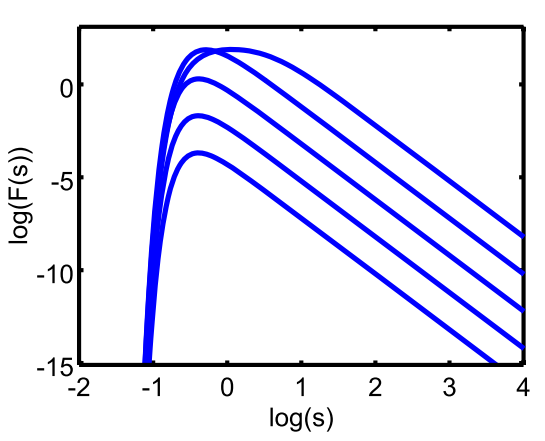

(b)
FIG. 1. (a) The integrand $F_{k}(t)$ is shown for different $k$ values in the range from 0.1 to 1000 corresponding to orbital energies of $\left[-0.005,-5 \times 10^{5}\right]$. (b) The corresponding integrands $F k(s)$ obtained by changing the variable $s=\frac{t}{k}$. 
TABLE I. The Hartree-Fock energy (in $E_{h}$ ) calculated for $\mathrm{H}_{2}, \mathrm{H}_{2} \mathrm{O}$, and $\mathrm{CO}$ using different grid sizes. The bond lengths $(\mathrm{R})$ and bond angle in degrees are given as footnote.

\begin{tabular}{lcccc}
\hline \hline & $\mathrm{H}_{2}{ }^{\mathrm{a}}$ & $\mathrm{H}_{2} \mathrm{O}^{\mathrm{b}}$ & $\mathrm{CO}^{\mathrm{c}}$ & $\mathrm{CO}^{\mathrm{d}}$ \\
\hline$h=0.05$ & -1.133629272 & -76.067474784 & -112.792666765 & -112.790874062 \\
$h=0.10$ & -1.133735078 & -76.067470421 & -112.792763416 & -112.790933269 \\
$h=0.15$ & -1.134032068 & -76.067244799 & -112.792867814 & -112.791386087 \\
$h=0.20$ & -1.130375595 & -76.071028544 & -112.789501793 & -112.790041365 \\
\hline Reference & $-1.133629571^{3,43}$ & $-76.067419^{44,45}$ & $-112.792463^{46}$ & $-112.79095^{47}$ \\
\hline \hline
\end{tabular}

${ }^{\mathrm{a}} \mathrm{R}=1.4$ bohr.

${ }^{\mathrm{b}} \mathrm{R}=1.80885$ bohr, $\mathrm{A}=104.52^{\circ}$.

${ }^{\mathrm{c}} \mathrm{R}=2.082100094$ bohr.

${ }^{\mathrm{d}} \mathrm{R}=2.312$ bohr.

Algorithm 1: Schematic description of the numerical self-consistent-field (SCF) algorithm.

while orbitals not converged do

Calculate the contributions to the Fock matrix and the metric as described in Ref. 42;

Diagonalize the general Fock matrix eigenvalue equation;

foreach orbital $i$ do Optimize orbital $i$ using the Helmholtz kernel

in Eq. (3);

end

\section{RESULTS}

The implementation was tested by performing calculations on $\mathrm{H}_{2}, \mathrm{H}_{2} \mathrm{O}$, and $\mathrm{CO}$ for which accurate benchmark results are available. The obtained Hartree-Fock energies are summarized in Table I. The dimension of the used Cartesian grid for the cube was $[-7,7]^{3}$ bohr $^{3}$, whereas the number of grid points varied from $73 \times 73 \times 73$ to $313 \times 313 \times 313$. The largest grid for $\mathrm{H}_{2}$ was $625 \times 625 \times 625$. The radius of the non-equidistant $1 \mathrm{D}$ grid of the bubbles was $20 \mathrm{bohr}$, which was divided to 6000 cells yielding 36001, 56341, and 60541 grid points for $\mathrm{H}, \mathrm{C}$, and $\mathrm{O}$, respectively.

The error in the total energy of $\mathrm{H}_{2}$ as a function of the grid spacings of the cube $(h)$ is shown in Figure 2. The energy of $\mathrm{H}_{2}$ in the basis-set limit can be estimated by extrapolation. By fitting the energies calculated using grid steps in the interval of $h=[0.02,0.08]$ to $E(h)=E_{0}+A h^{n}$, one obtains an extrapolated energy of $-1.133629609 E_{h}$ with an angular coefficient $n$ of 8.05. However, for grids denser than $h=0.05$, the accuracy

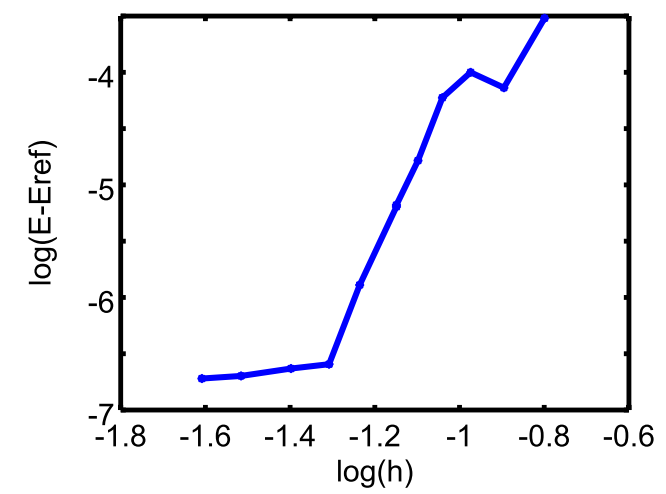

FIG. 2. The error in the Hartree-Fock energy for $\mathrm{H}_{2}$ calculated as a function of the grid spacing. is practically independent of the grid indicating that there are other factors than the Cartesian grid that limit the accuracy. For very sparse grids, whose spacings are larger than $h=0.2$, convergence problems appear in the self-consistent-field iteration procedure.

\section{CONCLUSIONS}

An integration of the Helmholtz kernel approach for optimizing orbitals in a self-consistent-field framework has been formulated in the double bubbles and cube basis. The algorithm is the final component for building a fully numerical quantum chemistry software for electronic structure calculations on general molecules at the Hartree-Fock level of theory. In the bubbles and cube approach, the steep functions in the vicinity of the nuclei are expanded in one-center functions, whereas the remaining part of the total functions, i.e., the cube part, is expanded on an equidistant three-dimensional grid.

The bubbles' part of the Helmholtz kernel is integrated by expanding the kernel in a series of spherical harmonics multiplied with modified spherical Bessel functions of the first and second kinds. The angular part can be integrated analytically, whereas the radial part is integrated using quadrature.

The use of equidistant grids simplifies the algorithms rendering efficient parallelization and the use of generalpurpose graphics processing units (GPGPU) feasible. The integration of the cube part of the Helmholtz kernel is performed using a similar method as that used for numerically integrating two-electron potentials implying that also a generalization of the fast multipole method (FMM) can be employed in order to speed up the integration of the Helmholtz kernel.

The numerical integration of the auxiliary dimension of the integral transformation of the Helmholtz kernel has been investigated in detail showing that a common integration grid can be used for molecules containing only light atoms. For heavier elements, a change in the integration variable can be employed yielding integrands of the same shape for all orbital energies.

The computational approach has been applied to $\mathrm{H}_{2}, \mathrm{H}_{2} \mathrm{O}$, and $\mathrm{CO}$ and the obtained energies have been compared to available literature data. The calculations show that our approach 
can reach the sub-milli- $E_{h}$ level accuracy corresponding to the ultimately desired chemical accuracy.

\section{ACKNOWLEDGMENTS}

This work was supported by the Academy of Finland through Project No. 275845. D.S. thanks Magnus Ehrnrooth Foundation, the Swedish Cultural Foundation in Finland, the Fulbright Foundation, and the Alexander von Humboldt Stiftung for financial support during his sabbatical leave. The computational resources were provided by CSC — the Finnish IT Center for Science.

${ }^{1}$ T. Yanai, G. I. Fann, Z. Gan, R. J. Harrison, and G. Beylkin, J. Chem. Phys. 121, 2866 (2004).

${ }^{2}$ T. Yanai, G. I. Fann, Z. Gan, R. J. Harrison, and G. Beylkin, J. Chem. Phys. 121, 6680 (2004).

${ }^{3}$ R. J. Harrison, G. I. Fann, T. Yanai, Z. Gan, and G. Beylkin, J. Chem. Phys. 121, 11587 (2004).

${ }^{4}$ J. Huang, J. Jia, and B. Zhang, Comput. Phys. Commun. 180, 2331 (2009).

${ }^{5}$ T. Yanai, G. I. Fann, G. Beylkin, and R. J. Harrison, Phys. Chem. Chem. Phys. 17, 31405 (2015).

${ }^{6}$ A. Cerioni, L. Genovese, A. Mirone, and V. A. Sole, J. Chem. Phys. 137, 134108 (2012).

${ }^{7}$ L. Frediani, E. Fossgaard, T. Flå, and K. Ruud, Mol. Phys. 111, 1143 (2013).

${ }^{8}$ S. R. Jensen, Real-Space All-Electron Density Functional Theory with Multiwavelets (UiT Norges Arktiske Universitet, 2014).

${ }^{9}$ S. Cools, B. Reps, and W. Vanroose, SIAM J. Sci. Comput. 36, B367 (2014).

${ }^{10}$ S. Cools and W. Vanroose, J. Comput. Phys. 308, 20 (2016).

${ }^{11}$ S. R. Jensen, T. Flå, D. Jonsson, R. S. Monstad, K. Ruud, and L. Frediani, Phys. Chem. Chem. Phys. 18, 21145 (2016).

${ }^{12}$ M. Kalos, Phys. Rev. 128, 1791 (1962).

${ }^{13}$ D. Sundholm, J. Chem. Phys. 122, 194107 (2005).

${ }^{14}$ J. Jusélius and D. Sundholm, J. Chem. Phys. 126, 94101 (2007).

${ }^{15}$ S. A. Losilla, D. Sundholm, and J. Jusélius, J. Chem. Phys. 132, 024102 (2010).

${ }^{16}$ S. A. Losilla and D. Sundholm, J. Chem. Phys. 136, 214104 (2012).

${ }^{17}$ E. A. Toivanen, S. A. Losilla, and D. Sundholm, Phys. Chem. Chem. Phys. 17, 31480 (2015).

${ }^{18}$ L. Plagne and J. Y. Berthou, J. Comput. Physics 157, 419 (2000).

${ }^{19}$ G. Fann, G. Beylkin, R. J. Harrison, and K. E. Jordan, IBM J. Res. Dev. 48, 161 (2004).

${ }^{20}$ F. A. Bischoff and E. F. Valeev, J. Chem. Phys. 134, 104104 (2011).
${ }^{21}$ D.I. Lyakh, Int. J. Quantum Chem. 114, 1607 (2014).

${ }^{22}$ M. Rakhuba and I. Oseledets, SIAM J. Sci. Comput. 37, A565 (2015).

${ }^{23}$ A. Durdek, S. R. Jensen, J. Juselius, P. Wind, T. Flå, and L. Frediani, Appl. Numer. Math. 92, 40 (2015).

${ }^{24}$ V. Khoromskaia, Comput. Methods Appl. Math. 14, 89 (2014).

${ }^{25}$ V. Khoromskaia and B. N. Khoromskij, Comput. Phys. Commun. 185, 2 (2014).

${ }^{26}$ V. Khoromskaia, D. Andrae, and B. N. Khoromskij, Comput. Phys. Commun. 183, 2392 (2012).

${ }^{27}$ B. N. Khoromskij, Chemom. Intell. Lab. Syst. 110, 1 (2012).

${ }^{28}$ B. N. Khoromskij, V. Khoromskaia, and H.-J. Flad, SIAM J. Sci. Comput. 33, 45 (2011).

${ }^{29}$ S. Goedecker and O. V. Ivanov, Solid State Commun. 105, 665 (1998).

${ }^{30}$ T. Yanai, R. J. Harrison, and N. C. Handy, Mol. Phys. 103, 413 (2005).

${ }^{31}$ H. J. Flad, W. Hackbusch, H. J. Luo, and D. Kolb, J. Comput. Phys. 205, 540 (2005).

${ }^{32}$ D. W. Massay, R. Acevedo, and B. R. Johnson, J. Chem. Phys. 124, 14101 (2006).

${ }^{33}$ C. D. Griffin, R. Acevedo, D. W. Massay, J. L. Kinsey, and B. R. Johnson, J. Chem. Phys. 124, 134105 (2006).

${ }^{34}$ H. Sekino, Y. Maeda, T. Yanai, and R. J. Harrison, J. Chem. Phys. 129, 34111 (2008).

${ }^{35}$ L. Genovese, A. Neelov, S. Goedecker, T. Deutsch, S. A. Ghasemi, A. Willand, D. Caliste, O. Zilberberg, M. Rayson, A. Bergman, and R. Schneider, J. Chem. Phys. 129, 014109 (2008).

${ }^{36}$ H. Sekino, A. Matsumura, Y. Yokoi, and T. Kato, Int. J. Wavelets Multiresolution Inf. Process. 11, 1360008 (2013).

${ }^{37}$ J. Pipek and S. Nagy, J. Comput. Chem. 34, 460 (2013).

${ }^{38}$ M. Bachmayr, SIAM J. Numer. Anal. 51, 2491 (2013).

${ }^{39}$ H. Sekino, Y. Yokoi, and R. J. Harrison, J. Phys.: Conf. Ser. 352, 012014 (2012).

${ }^{40}$ Dover Books on Advanced Mathematics, Corrected ed., edited by M. Abramowitz and I. A. Stegun (Dover, New York, 1965).

${ }^{41}$ P. Parkkinen, S. A. Losilla, E. Solala, E. A. Toivanen, W.-H. Xu, and D. Sundholm, J. Chem. Theory Comput. 13, 654 (2017).

${ }^{42}$ S. A. Losilla, M. A. Watson, A. Aspuru-Guzik, and D. Sundholm, J. Chem. Theory Comput. 11, 2053 (2015).

${ }^{43}$ A. V. Mitin, Phys. Rev. A 62, 010501 (2000).

${ }^{44}$ T. Helgaker, W. Klopper, H. Koch, and J. Noga, J. Chem. Phys. 106, 9639 (1997).

${ }^{45}$ A. Halkier, T. Helgaker, P. Jørgensen, W. Klopper, H. Koch, J. Olsen, and A. K. Wilson, Chem. Phys. Lett. 286, 243 (1998).

${ }^{46}$ K. A. Peterson and T. H. Dunning, J. Mol. Struct.: THEOCHEM 400, 93 (1997).

${ }^{47}$ L. Laaksonen, P. Pyykkö, and D. Sundholm, Comput. Phys. Rep. 4, 315 (1986). 